\title{
L'utilisation par la viticulture française d'un exercice de prospective pour l'élaboration d'une stratégie d'adaptation au changement climatique
}

\section{Using foresight exercise to design adaptation policy to climate change: The case of the French wine industry}

P. Aigrain ${ }^{1}$, B. Bois ${ }^{8}$, F. Brugiere ${ }^{1}$, E. Duchene ${ }^{7}$, I.G. de Cortazar-Atauri ${ }^{6}$, J. Gautier ${ }^{2}$, E. Giraud-Heraud ${ }^{5}$, R. Hammond $^{4}$, H. Hannin ${ }^{3}$, N. Ollat ${ }^{5}$, et J.M. Touzard ${ }^{4}$

${ }^{1}$ FranceAgriMer Montreuil, France

2 INAO Montreuil, France

${ }^{3}$ Univ Montpellier, MOISA, Montpellier SupAgro/IHEV, Montpellier, France

${ }^{4}$ Univ Montpellier, Innovation, INRA, Montpellier, France

${ }^{5}$ EGFV, ISVV-INRA Bordeaux, France

${ }^{6}$ Agroclim, INRA Avignon, France

${ }^{7}$ SVQV, INRA Colmar, France

${ }^{8}$ Université de Bourgogne Dijon, France

Foresight studies are regularly conducted at sectoral or geographical scales, in order to help policy makers and economic actors to define their strategy of adaptation to climate change $(C C)$.

Some studies are rather "quick exercises", in which a panel of experts is consulted to define the expected impacts of CC and to identify adaptation leviers for future policy. In other cases, a true foresight methodology is developed, leading to the building of scenarios based on : i) a systemic and participatory approach, ii) the definition of key variables, iii) the choice of assumptions and the coherent relations between these assumptions, the narrative description of scenarios.

This participatory dimension of the approach may be relevant to better integrate the climate change challenge into future strategies chosen by decision makers.

Such an exercise was conducted in the framework of the multidisciplinary INRA / LACCAVE research project (2013-2017) to consider the CC adaptation strategy that could be implemented in the French wine industry by 2050.

This communication presents the outcomes and insights opened up by this prospective exercise, based on a new approach derived from the scenarios method, following two main steps :

1) The first step consists of a "classical", topdown, expert-led prospective approach, including the selection of four possible adaptation scenarios ; it was presented at the OIV Congress in Bento Gonçalves in 2016.

2) The second step is based on a bottom-up participatory approach with regional and national stakeholders during six regional meetings. Each meeting brought together 60 to 100 actors invited to interact on four scenarios and on solutions that could be developed : the "Conservative scenario" driven by incremental changes; the "Innovative scenario" driven by the development of a wide range of technical innovations ; the "Nomad scenario" focusing on the relocation of vineyards ; the "Liberal scenario" where "everything would be possible anywhere”.

A national working group has been set up by INAO ${ }^{1}$ and FranceAgriMer ${ }^{2}$, inviting the main national wine organizations to analyze the information collected during the regional meetings. This working group aims at making proposals to design the future climate strategy of the sector, taking into account the inter-regional diversity of situations.

The participants expressed common positions (perception of limited resilience of the wine industry, increasing need for innovation, opposition to the relocation of vineyards...), but regional specificities were also noted (for example : development of irrigation in the south of France or calls for new research on pest control in other French regions). The large majority of stakeholders wanted to favor the pathway to the innovative scenario, with several regional variations.

\footnotetext{
${ }^{1}$ Institut National de l'Origine et de la Qualité.

2 'Établissement national des produits de l'agriculture et de la mer.
} 
This foresight exercise generated a greater stakeholder awareness of the CC issue, leading to the implementation of collective actions at different scales. Its findings will provide significant support to the wine industry in developing a national climate strategy, taking into account regional specificities.

\section{Rappel introductif : la réalisation d'une prospective pour le secteur vigne et vin dans le contexte du changement climatique}

A l'horizon 2050, la filière vitivinicole française va devoir s'adapter au changement climatique, qui modifiera à la fois les conditions de production des vins, leurs caractéristiques organoleptiques et leurs marchés. Par ailleurs, d'autres facteurs tels que les évolutions des préoccupations de santé et d'environnement, les changements géopolitiques ou technologiques, les nouvelles conditions de consommation $\mathrm{du}$ vin ou encore les modifications de politique agricole affecteront le secteur vitivinicole à cette échéance.

De nombreux leviers d'adaptation peuvent être envisagés, comme l'introduction de nouvelles variétés ou des modifications des pratiques viticoles et œnologiques, mais aussi de nouvelles localisations, la réorganisation de la filière et de son cadre réglementaire, ou encore la redéfinition des politiques de recherche et d'innovation (Jones, Webb, 2010 ; Ollat, Touzard, van Leuwen, 2016).

Si de telles actions ont déjà été mises en œuvre ou sont envisagées, cela a souvent été fait sans vision très claire, sans réflexion collective sur des futurs possibles affectés par le changement climatique. Engager une large réflexion, imaginer des futurs possibles devient alors stratégique pour les acteurs de la vigne et du vin, ainsi que pour la Recherche amenée à les accompagner.

Le projet $\mathrm{LACCAVE}^{3}$ a été mis en place par l'INRA entre 2012 et 2016 pour étudier les impacts du changement climatique et les adaptations potentielles $\mathrm{du}$ secteur viti-vinicole français (Ollat, Touzard, 2014). Il a associé des chercheurs de différentes disciplines (climatologie, génétique, agronomie, œnologie, économie, sociologie...), issus de 23 équipes de l'INRA, du CNRS, d'universités et d'écoles d'ingénieurs. Une des opérationsphares du projet LACCAVE a été la conduite d'un exercice de prospective, avec plusieurs objectifs :

- identifier et explorer différentes stratégies d'adaptation pour la viticulture française dans un contexte de changement climatique, à l'horizon 2050 ;

- tester une méthode pour élaborer des «chemins» favorisant la mise en cuvre de ces stratégies d'adaptation ;

- développer une vision commune et des pratiques de travail en réseau entre chercheurs et organisations de la filière.

\section{Une méthode originale de prospective}

En partant des travaux du projet LACCAVE et d'études de terrain préalables dans différents vignobles français,

\footnotetext{
${ }^{3}$ Projet LACCAVE : Projet de recherche interdisciplinaire centré sur l'adaptation au changement climatique pour la vigne et le vin.
}

un groupe pluridisciplinaire de chercheurs et d'experts issus d'organismes publics de la filière viti-vinicole française (INAO et FranceAgriMer) a présélectionné quatre scénarios d'adaptation de la filière viticole française au changement climatique. Ces scenarios sont considérés à l'horizon 2050 en retenant comme hypothèse de travail le scenario climatique médian du GIEC. Ils peuvent être représentés en croisant deux dimensions majeures de l'adaptation : les choix de localisation des vignes et l'ampleur de l'innovation technologique. Chaque scénario correspond donc au développement d'une stratégie dominante sur une trentaine d'années (Aigrain et al., 2016a, c) :

- une stratégie «conservatrice» où changements technologiques et géographiques sont limités,

- une stratégie «nomade» où le déplacement des vignobles est le vecteur d'adaptation privilégié,

- une stratégie «innovante» où l'adaptation se fait par l'intégration d'innovations sans relocalisation majeure du vignoble,

- une stratégie "libérale" largement ouverte aux changements de toute nature.

Les chemins permettant le développement de ces stratégies ont été précisés et décrits en retenant et combinant soixante hypothèses concernant différents domaines qui influencent l'évolution du « système vigne et vin »:

- le contexte général de ce système avec en particulier la prise en compte des politiques publiques et de leurs évolutions ;

- le contexte viti-vinicole international et notamment l'évolution des règles internationales ;

- le contexte viti-vinicole local et national avec notamment les relations filière/recherche, la capacité structurelle d'adaptation des exploitations ou encore l'évolution des attitudes des consommateurs ;

- les conséquences sur la filière, incluant l'évolution de sa gouvernance ou l'évolution du poids des indications géographiques.

\section{Une mobilisation intense et croissante à toutes les échelles}

Au plan international, le projet LACCAVE anticipe et souhaite stimuler une mobilisation sur la question du changement climatique en viticulture. Ses travaux et ses résultats méritent d'être communiqués à l'international, en particulier dans le cadre des groupes d'experts de l'OIV et dans des instances connexes comme le congrès mondial de la vigne et du vin. La problématique du changement climatique en viticulture est en effet abordée à l'OIV de façon croissante et depuis plusieurs années. La France devrait pouvoir jouer un rôle moteur dans la mise en place d'une réflexion internationale sur l'adaptation au changement climatique en viticulture et sur la contribution de ce secteur à la réduction des émissions de gaz à effet de serre. Une présentation des travaux de la démarche prospective a été effectuée fin 2016 au congrès mondial de la vigne et du vin à Bento Gonçalves (Aigrain et al., 2016 b, c). Le présent papier prend en compte les travaux conduits et les avancées observables depuis lors. 
Au plan national, la genèse d'un groupe conjoint créé par l'INAO et FranceAgriMer (GT FAM/INAO) sur cette thématique mérite d'être mentionnée.

Suite aux présentations internationales de 2016, Jérôme DESPEY et Christian PALY respectivement présidents du Conseil spécialisé Vin de France AgriMer et du Comité national des AOC de l'INAO, ont souhaité que la filière française puisse s'approprier cette thématique en relation avec la communauté scientifique.

Début 2017, ils ont mis en place un groupe de travail conjoint sur cette thématique, présidé par Bernard ANGELRAS, afin notamment de :

- valoriser au sein de la profession les travaux scientifiques mis en place depuis plusieurs années et en faciliter l'appropriation ;

- permettre à la filière d'anticiper les conséquences du changement climatique et de devenir un acteur de son avenir en travaillant sur l'élaboration d'une stratégie nationale, incluant des adaptations spécifiques dans les différentes régions ; en particulier cette stratégie devrait inclure les moyens nécessaires pour :

- construire les demandes de la filière adressées à la Recherche

- anticiper les besoins d'évolution réglementaire pour l'adaptation au changement climatique.

Au plan régional, six réunions se sont tenues dans les principaux vignobles français ${ }^{4}$ :

La présente publication rend compte des méthodes mobilisées et des résultats obtenus lors de ces réunions régionales, et de leur consolidation pour l'élaboration d'une stratégie nationale.

\section{Le choix d'une méthode participative pour mobiliser la filière}

L'enjeu de la restitution d'un travail en prospective est d'amener un public qui n'a pas été impliqué dans l'élaboration des scénarios prospectifs à en comprendre le statut et à accepter de se projeter dans les futurs proposés pour en envisager les enjeux et les conséquences et pour ensuite décider de leur caractère souhaitable ou redoutable, sans se positionner sur leur véracité ou leur probabilité d'advenue (Few et al., 2011 ; Godet, Durance, 2011). Une restitution publique réunissant en un même lieu différents acteurs de la filière et de la recherche est une occasion précieuse qui peut être utilisée pour permettre des échanges intenses, sans enjeux immédiats, des temps de partage de points de vue créateurs d'une vision commune, dans une atmosphère studieuse et propice à l'expression de tous.

Le vignoble français étant fortement structuré autour des indications géographiques et les incidences des évolutions du climat étant potentiellement différentes d'une région viticole à l'autre, il a été décidé d'organiser des forums de mises en débat de ces résultats dans différents vignobles régionaux.

L'organisation de chaque réunion régionale a été prise en charge par un organisme de recherche (centre INRA

\footnotetext{
${ }^{4}$ Projet LACCAVE : Projet de recherche interdisciplinaire centré sur l'adaptation au changement climatique pour la vigne et le vin.
}

ou Université), et/ou par les interprofessions locales. Dans chaque région, les organisations viti-vinicoles représentées localement ont été invitées à relayer l'information et les objectifs de la journée et à proposer un certain nombre de participants. L'information a également circulé par le bouche à oreille et certaines personnalités ont été invitées spécifiquement par l'équipe d'animation.

Le nombre de participants était dans tous les cas limité à 100 par journée. A l'inscription, chaque participant était invité à préciser ses «titres et qualités» et son principal secteur d'activité.

Ces informations ont été utilisées par les organisateurs pour constituer des groupes de travail de 6 à 8 personnes aux profils les plus variés possibles et pour désigner, parmi les participants, un responsable du groupe. Le jour du forum, les groupes ont fonctionné par table, chacun étant équipé d'une tablette (type i-pad) programmée pour faciliter la saisie par le responsable du groupe, des éléments échangés et des votes individuels.

Chaque forum s'est déroulé selon le même protocole. Après une introduction précisant des généralités sur le changement climatique et la méthode d'élaboration des scénarios d'adaptation, chaque chemin conduisant à un scénario a été présenté successivement aux participants. Des présentateurs différents pour chaque séquence avaient été préalablement désignés au sein de l'équipe d'animation. Après la présentation de chaque chemin, il était demandé aux participants de se projeter en 2050 et d'échanger au sein de chaque table pendant une vingtaine de minutes sur les enjeux et les conséquences, pour leur région. Ces discussions collectives s'organisaient selon les aspects techniques et de marché, mais aussi liés aux acteurs, aux territoires et à organisation de la filière, en qualifiant leurs contributions comme positive, neutre ou négative pour la filière viticole. Toutes les contributions étaient ensuite transmises à un PC central et regroupées dans un tableur. L'information recueillie était alors immédiatement traitée par l'équipe d'animation pour en établir une synthèse de façon à mettre ces contributions en débat général en seconde partie du forum. Ainsi, en moins de deux heures, tous les participants ont exploré, critiqué, reformulé et surtout débattu en petits groupes des chemins conduisant aux quatre archétypes de stratégie d'adaptation.

Dans la seconde partie de la journée, les synthèses des contributions de l'ensemble des participants sur les enjeux et conséquences, établies en séance, ont été présentées aux participants par grand thème (techniques, segment de marché etc.) et par scénario d'adaptation. Les participants ont également été amenés à réagir en plénière sur ces synthèses. Puis après une brève introduction sur la notion d'attitude stratégique (proactivité positive, négative, réactivité anticipée, veille), chaque participant a été amené à se prononcer par vote électronique sur l'attitude stratégique privilégiée pour chaque scénario. En parallèle, et une fois l'attitude stratégique choisie pour chaque scénario, chaque participant a pu proposer sur papier des leviers d'action à mettre en œuvre. Le résultat des votes a été immédiatement révélé aux participants et commenté par les animateurs. Chaque vote a été suivi d'un débat général et d'une conclusion de la journée par un grand témoin professionnel. L'information recueillie sur papier a fait l'objet d'un traitement ultérieur. Les propositions ont été saisies intégralement puis catégorisées, constituant une 
base de données où les propositions peuvent être croisées par région, attitude stratégique sur chaque scénario et domaine d'action.

\section{Résultats obtenus}

Les résultats sont présentés et analysés comme suit :

- les participants,

- les perceptions des différents chemins,

- les attitudes stratégiques votées par les participants,

- les leviers d'actions proposés pour chaque couple attitude stratégique/scenario.

\subsection{Les participants :}

Sur un total de 355 participants dont les données de signalétique sont exploitables, un tiers déclare intervenir au niveau de la production (et parmi eux $2 / 3$ sont des chefs d'exploitation), un autre tiers se positionne sur les activités de Recherche, conseil, expérimentation et R\&D. Dix \% des participants travaillent dans l'administration et presque autant $(8 \%)$ dans les structures professionnelles de la filière. Les pépiniéristes d'une part et les négociants d'autre part se sont peu mobilisés (avec respectivement 2 et 20 participants). Le manque d'information et la durée annoncée de la réunion (une journée) a pu représenter pour certains un frein à la participation. Néanmoins l'objectif de mixité «professionnels de la filière vignes et vins » / « recherche et administration » peut être considéré comme atteint.

\subsection{Analyse des perceptions des scenarios d'adaptation}

\section{a) Selon les régions}

Les perceptions des différents scénarios d'adaptation, résumées en séance, révèlent des sensibilités régionales contrastées. Même si une certaine homogénéité peut apparaitre dans la perception globale du changement climatique et des enjeux et conséquences des scénarios, des différences entre régions peuvent être soulignées. $\mathrm{Si}$ tous les participants sont convaincus de l'enjeu que représente le changement climatique, le caractère d'urgence et de menace est plus marqué dans les zones méridionales. Cela a pu conduire à une plus faible distinction entre scénarios et à une vision pessimiste quel que soit le futur envisagé. En fonction des spécificités régionales, un élément marquant ressort de chaque journée. Le désarroi est plus marqué dans une région méridionale fortement structurée autours des AOC (Avignon), alors que l'exploration de solutions nouvelles ressort comme une opportunité pour la région de Montpellier où les modèles économiques sont plus diversifiés. La Champagne, où la filière est très organisée autour d'une interprofession, craint la remise en cause de cette spécificité particulièrement performante sur le plan technique et économique. En Bourgogne et Beaujolais, c'est la tension sur le foncier qui apparait comme la préoccupation majeure. A Bordeaux et dans le Sud-Ouest, des aspects positifs du changement climatique (CC) sont identifiés pour tous les scénarios et l'accent est mis sur l'importance future du négoce et l'évolution des qualités.
En Alsace, l'attachement au modèle socio-technique actuel est marquante, avec la motivation de modifier les pratiques dans le cadre autorisé et actuel pour préserver ce modèle.

\section{b) Des perceptions nuancées sur les différents chemins}

Le chemin vers le scénario conservateur est perçu comme comprenant un certain nombre de conséquences plutôt positives parce qu'il impose de valoriser au maximum les possibilités existantes : la diversité des clones et des variétés (avec de nouveaux porte-greffes), les effets millésime dans les types de vins, la diversité de gamme et des circuits... Il permet de renouer avec les consommateurs par la mise en avant de l'environnement, notamment via l'œnotourisme, les liens de proximité ou les services environnementaux (lutte contre les incendies, stockage de $\mathrm{CO}^{2} \ldots$ ). Il impose particulièrement la professionnalisation et des initiatives de la part des organisations viticoles pour assurer un minimum de R\&D, de conseil, de veille sanitaire, de suivi du CC. Il s'appuie sur le travail de groupes locaux et l'aide des collectivités...

En parcourant ce chemin, la viticulture française reste une référence, autour de vins de terroir élitistes amenant un renouvellement des acteurs limité.

Des conséquences négatives sont également mises en avant, à commencer par la baisse des rendements et un accroissement de la variabilité qualitative du vin qui affectent la rentabilité de nombreuses exploitations (avec des différences régionales). Les liens avec la recherche sont faibles, peu d'avancées techniques majeures sont développées, l'irrigation est de plus en plus contrainte.

Les vins français, élitistes et chers, sont présents sur des marchés étriqués et incertains. Ils ont disparu des entrées de gamme. Progressivement, la viticulture française perd de son importance, de sa compétitivité et de ses emplois.

Le chemin vers le scénario nomade fait écho à des visions largement médiatisées de l'impact du changement climatique comme «la production d'effervescents concurrents du champagne en Suède ou en Angleterre», qui stimulent des échanges entre les participants. En positif, ce scénario est vu comme favorisant l'exploration de nouvelles combinaisons sol / climat / matériel végétal/ pratiques, le «retour» à la polyculture voire l'invention de nouvelles formes de viti-écologie (soft innovations). On observe de nouvelles manières d'associer qualité et territoire : les nouveaux vignobles étendent l'offre de vins, ouvrent de nouveaux marchés. Avec de nouveaux acteurs (investisseurs, agriculteurs, urbains), la filière oscille entre dynamisme et prise de risques sur de nouveaux territoires. Dans ce chemin, l'irrigation a pu se développer, même si la concurrence sur l'eau et l'espace est vive.

En négatif, ce chemin conduit à la fragilisation des vignobles actuels et des savoir-faire locaux, de l'œnotourisme, des paysages, des liens à la gastronomie... Ceux qui ne «bougeront pas» verront les risques accentués et subiront une variabilité accrue des caractéristiques des vins. Les nouveaux vignobles sont imaginés «industriels», en opposition avec de «petits producteurs de vins élitistes». Ce chemin sonne la fin des appellations actuelles (AOP, IGP) et de leur gouvernance 


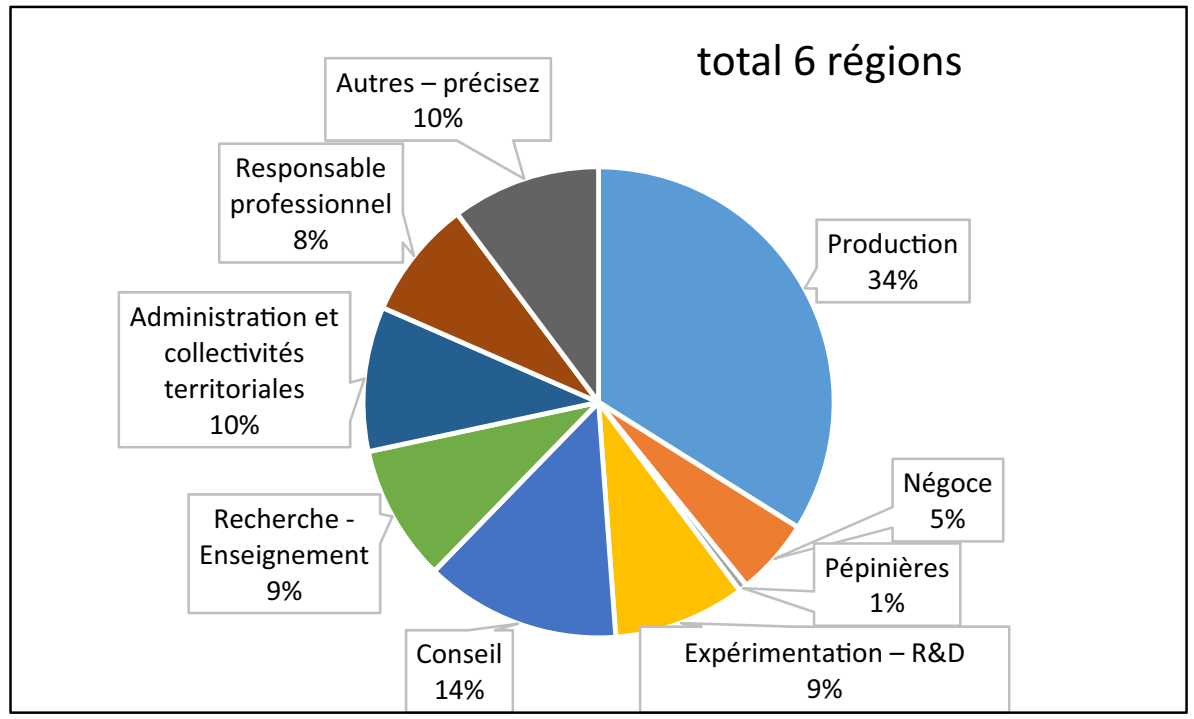

Figure 1. Répartition des participants par type d'activité.

(ODG, interprofessions, INAO) avec une concentration des opérateurs autour des marques et un fort développement des activités de conseil et de contrôle privés.

Le chemin vers le scénario libéral, interroge plus fortement encore les valeurs de la filière vignes et vins française. Néanmoins, un certain nombre de points positifs y sont décelés. Sur ce chemin, la $R \& D$ est soutenue par le secteur privé, plutôt vu comme pouvant se saisir des opportunités avec réactivité en lien étroit avec les attentes des consommateurs et des investisseurs. D'où un foisonnement de nouveautés : nouveaux cépages, irrigation, robotisation, chimie et marketing stabilisent les qualités et les rendements, nouveaux «vins», nouveaux marchés, nouveaux consommateurs et nouveaux métiers, nouveaux investisseurs... Et tout cela dans un contexte de réduction de charges et de contraintes administratives. Mais les aspects négatifs dominent : la privatisation de la R\&D exclut les petits producteurs ou les petits vignobles qui ne peuvent se la payer et conduit à une viticulture à 2 vitesses avec des vins de niche vs des vins industriels. Cette industrialisation du vin conduit à des pertes de diversité et typicité avec des «vin éprouvette», mouillés, aromatisés, ... Normes et contrôles ayant été libéralités, la disparition des AOP et des IGP est programmée. Les coteaux sont abandonnés, la production se concentre. Les producteurs perdent le pouvoir au profit de pôles industriels et de négoce. La filière est désorganisée.

Enfin, le chemin vers le scénario innovant semble d'emblée plus séduisant. Néanmoins des risques sont identifiés : ce chemin s'accompagne de besoins de financement (investissement, assurance), qui induiront une hausse des coûts et pas forcément d'accès à tous à l'innovation. Il faudra faire entrer de nouveaux investisseurs, de nouveaux acteurs qui peuvent marginaliser des viticulteurs et des vignobles de petite taille. Robots, NTIC, innovation œnologique ont pu réduire diversité et référence à l'histoire (avis divergent sur ce point). L'adoption des innovations risque d'affaiblir les AOP actuelles au profit des IGP voire des VSIG. Mais les visions positives prévalent. Nouveaux cépages et porte greffes, œnologie «corrective», nouvelles technologies au vignoble... ont permis de maintenir globalement les vignobles en place. La coexistence de plusieurs modèles viticoles et de vins est autorisée : viticulture de précision, agriculture biologique/agroécologie, AOP innovants, vins sans pesticide, nouveaux produits. L'innovation va audelà de la vigne et de la cave : innovations de packaging, commerciales, de service (assurance, big data..). Les cahiers des charges des indications géographiques sont modifiés. Le rôle des interprofessions s'accroit parallèlement à une ouverture de la gouvernance, l'innovation bénéficie de soutiens publics, et la gestion du foncier/patrimoine devient une question centrale (protection, ressources...).

\subsection{Attitudes stratégiques : la voie de l'innovation préférée partout ... . mais avec quel contenu?}

Après la présentation de la synthèse des contributions sur les enjeux et conséquences des scénarios, il a été demandé aux participants de s'exprimer sur les attitudes stratégiques à adopter vis-à-vis des chemins conduisant à ces scénarios en choisissant parmi 5 attitudes possibles :

- Proactivité positive : agir dès aujourd'hui pour favoriser l'advenue de ce futur possible ;

- Proactivité négative : agir dès aujourd'hui pour défavoriser l'advenue de ce futur possible ;

- Réactivité anticipée : se préparer dès aujourd'hui à l'advenue de ce futur ;

- Veille : Cette possibilité doit être placée sous surveillance, pour savoir si son advenue se dessine au fur et à mesure du temps ;

- Aucune attitude : cette histoire ne présente pas d'intérêt particulier.

Le tableau ci-après est une compilation des résultats obtenus dans les 6 régions.

Les résultats de ces votes (Tableau 1) doivent être pris avec précaution (a priori positif et négatif sur certains chemins, connotation positive ou négative de leur dénomination, participants plus ou moins sensibilisés au $\mathrm{CC}$ et à l'innovation...) mais ils sont très marqués et expriment une tendance réelle. 
Tableau 1. \% des votants pour chaque attitude stratégique.

\begin{tabular}{|l|l|l|l|l|l|}
\cline { 2 - 6 } \multicolumn{1}{c|}{} & $\begin{array}{l}\text { proactivité } \\
\text { positive }\end{array}$ & $\begin{array}{l}\text { proactivité } \\
\text { négative }\end{array}$ & $\begin{array}{l}\text { réactivité } \\
\text { anticipée }\end{array}$ & veille & $\begin{array}{l}\text { aucun } \\
\text { intérêt }\end{array}$ \\
\hline chemin vers la stratégie conservatrice & $20 \%$ & $32 \%$ & $28 \%$ & $16 \%$ & $4 \%$ \\
\hline chemin vers la stratégie innovante & $\mathbf{7 2} \%$ & $2 \%$ & $23 \%$ & $2 \%$ & $1 \%$ \\
\hline chemin vers la stratégie nomade & $3 \%$ & $39 \%$ & $28 \%$ & $28 \%$ & $2 \%$ \\
\hline chemin vers la stratégie libérale & $4 \%$ & $\mathbf{5 7} \%$ & $17 \%$ & $20 \%$ & $2 \%$ \\
\hline
\end{tabular}

Tableau 2. Répartition des attitudes stratégiques par région.

\begin{tabular}{|c|c|c|c|c|c|}
\hline \multicolumn{2}{|c|}{ vote tablette en $\%$} & «Conservatrice » & «Innovatrice » & « Nomade » & « Libérale » \\
\hline \multirow{5}{*}{$\begin{array}{l}\text { Proactivité } \\
\text { Positive }\end{array}$} & Avignon & 16.9 & 63.6 & 2.6 & 1.3 \\
\hline & Alsace & 29.3 & 56.1 & 0.0 & 2.5 \\
\hline & Bordeaux & 13.3 & 81.7 & 1.6 & 5.0 \\
\hline & Champagne & 29.4 & 66.7 & 2.0 & 3.9 \\
\hline & Languedoc $\mathrm{R}$ & 23.9 & 81.7 & 4.3 & 5.6 \\
\hline \multirow{4}{*}{$\begin{array}{l}\text { Réactivité } \\
\text { Anticipée }\end{array}$} & Bordeaux & 36.7 & 16.7 & 33.3 & 21.7 \\
\hline & Bourgogne & 20.0 & 15.0 & 32.5 & 15.0 \\
\hline & Champagne & 21.6 & 29.4 & 11.8 & 9.8 \\
\hline & Languedoc R & 18.3 & 14.1 & 39.1 & 21.1 \\
\hline \multirow[t]{3}{*}{ Veille } & Avignon & 7.3 & 0.0 & 26.3 & 14.5 \\
\hline & Alsace & 17.1 & 4.9 & 26.8 & 12.5 \\
\hline & Languedoc $\mathrm{R}$ & 28.2 & 2.8 & 23.2 & 21.1 \\
\hline \multirow{6}{*}{$\begin{array}{l}\text { Proactivité } \\
\text { Négative }\end{array}$} & Avignon & 44.2 & 6.5 & 36.8 & 71.1 \\
\hline & Alsace & 14.6 & 0.0 & 61.0 & 60.0 \\
\hline & Bordeaux & 23.3 & 1.6 & 26.7 & 43.3 \\
\hline & Bourgogne & 47.5 & 0.0 & 35.0 & 50.0 \\
\hline & Champagne & 43.1 & 2.0 & 58.8 & 66.7 \\
\hline & Languedoc R & 21.1 & 1.4 & 31.9 & 52.1 \\
\hline
\end{tabular}

Le vote «équilibré» sur le chemin conservateur témoigne de positions variables sur la situation actuelle et sa résilience vis-à-vis du CC. Cela dépend des régions (plus ou moins impactées et compétitives : alsace et champagne plus favorables), des catégories d'acteurs (viticulteurs plus favorables) et du niveau de satisfaction au regard de l'état actuel de l'activité...

Le chemin vers le scénario nomade est certes rejeté mais surtout il interroge, et doit être «surveillé»: est-il possible ? De nouveaux vignobles peuvent-ils réellement se développer?

Le rejet du chemin libéral est très fort, car associé à une perte d'influence des viticulteurs, couplé à une redéfinition du vin, à un bouleversement de tous les repères ...

Le vote en faveur du chemin innovant exprime un message clé : innover pour rester, pour préserver les investissements individuels et collectifs réalisés dans le territoire (patrimoine, image, autres activités, liens sociaux...) et qui créent la valeur du vin... mais jusqu'où ?

L'analyse par région (Tableau 2) fait ressortir plusieurs orientations :

Globalement, si toutes les régions mettent en avant la stratégie innovante, on note que les régions septentrionales (Alsace et Champagne) écartent moins que les autres la stratégie conservatrice.
Contrairement à certaines idées reçues, ce sont les régions telles que Bordeaux et Bourgogne/Beaujolais qui rejettent le plus la stratégie conservatrice en faveur de la stratégie innovante, rejoignant en cela une des régions méridionales (Montpellier).

L'Alsace est originale dans son attitude stratégique face à la stratégie innovante en mettant en avant une part non négligeable de réactivité anticipée.

Face à la stratégie nomade, on constate encore le particularisme entre les régions les plus septentrionales (Alsace et Champagne) qui veulent tout mettre en œuvre pour éviter qu'elle devienne réalité, alors que les autres régions adoptent une attitude partagée entre agir pour éviter sa mise en place et se préparer à ce qu'elle advienne.

Le rejet de la stratégie libérale est général, mais il l'est moins à Bordeaux, en Bourgogne et en Languedoc qu' ailleurs.

\subsection{Analyse des leviers d'action proposés}

Au total sur les 342 votants des 6 régions, 328 (96\%) ont exprimé des propositions de leviers d'action pour mettre en œuvre leur option stratégique pour chaque chemin. 2222 propositions ont été ainsi formulées, de nombreux participants en ayant exprimé plusieurs par chemin.

Parmi ces propositions (Tableau 3), $25 \%$ peuvent être considérées comme des commentaires ou des orientations 
Tableau 3. Nombre et \% d'acteurs et de propositions par type d'action.

\begin{tabular}{|l|l|l|l|l|}
\hline $\begin{array}{l}\text { types } \\
\text { d'action }\end{array}$ & Nb Acteurs & \%Acteurs & Nb propos & \%propos \\
\hline Etat & 273 & $\mathbf{8 3}$ & 937 & $\mathbf{4 2}$ \\
\hline Collectif & 303 & $\mathbf{9 2}$ & 1384 & $\mathbf{6 2}$ \\
\hline Individus & 188 & $\mathbf{5 7}$ & 433 & $\mathbf{1 9}$ \\
\hline Mixte & 248 & $\mathbf{7 6}$ & 680 & $\mathbf{3 1}$ \\
\hline Généralités & 222 & $\mathbf{6 8}$ & 559 & $\mathbf{2 5}$ \\
\hline Total & 342 & $\mathbf{1 0 0}$ & 2222 & $\mathbf{1 0 0}$ \\
\hline
\end{tabular}

très générales, comme par exemple «il faut défendre les AOC», «renforcer le rôle des syndicats» ou «mettre de la souplesse dans le système». $75 \%$ des propositions correspondent par contre à des actions plus concrètes, portées avant tout par l'action collective seule (62\%) ou combinant action collective et intervention publique (31\%). $19 \%$ se réfèrent directement à une action individuelle, souvent proposée par un viticulteur envisageant par exemple de «planter des cépages tardifs» ou de «développer un nouveau vin». $12 \%$ des actions invoquent l'action publique seule, surtout pour des questions de réglementation ou de recherche fondamentale.

a) Présentation des principaux leviers exprimés par couple Chemin/Attitude stratégique

\section{Le chemin vers le scénario conservateur exprime deux attitudes opposées :}

Certains veulent le favoriser et proposent de :

- développer une $\mathrm{R} \& \mathrm{D}^{5}$ avec les viticulteurs sur les enjeux environnement et terroirs,

- expérimenter des pratiques agroécologiques, valoriser savoir-faire locaux et diversité,

- accompagner le développement de cépages anciens ou originaires d'autres régions,

- communiquer sur les vins actuels, leurs liens au terroir, les effets climatiques.

D'autres veulent l'éviter et proposent de :

- promouvoir la R\&D et l'innovation chez les viticulteurs et les décideurs,

- informer sur les vulnérabilités des vignobles face au changement climatique,

- soutenir les changements techniques et la révision des cahiers des charges.

\section{Plébiscitée, la stratégie innovante est vue comme un moyen pour maintenir une viticulture liée aux terroirs et organisée}

Pour la favoriser les participants proposent de :

- Favoriser partout R\&D, formation et veille : cépages résistants, irrigation, désalcoolisation, mécanisation, nouveaux produits... pour des innovations adaptées localement

- Accompagner le renouvellement de l'encépagement à la fois vers la viticulture et l'œnologie de précision, avec ou sans irrigation, et vers l'agriculture biologique

\footnotetext{
${ }^{5}$ Recherche et Développement.
}

- Ecouter, informer, éduquer les consommateurs : investir dans le marketing sur les vins du changement climatique et l'acceptation des nouvelles technologies

- Renforcer les organisations collectives pour soutenir l'innovation, maintenir les localisations et le modèle familial, contrôler concentrations et investisseurs extérieurs.

\section{Le rejet du chemin Nomade est motivé par la crainte d'un effacement des terroirs, paysages et typicités des vins}

Pour l'éviter les participants proposent de : Renforcer les $\mathrm{ODG}^{6}$ et étudier les terroirs existants et leurs périphéries

- Appuyer les actions collectives, syndicales, politiques pour défendre les terroirs, l'identité des produits, éviter les dérégulations, être vigilants...

- Sensibiliser les consommateurs et développer une communication autour des terroirs et des apports de la filière, au-delà du vin : emplois, paysages, économie, culture

- Développer la recherche et les innovations pour le maintien des terroirs actuels : cépages et portegreffe, irrigation... Innover dès aujourd'hui pour éviter ce scenario.

\section{L'opposition est forte vis-à-vis de la stratégie libérale, perçue comme remettant en cause les bases de la viticulture française}

Pour l'éviter les participants proposent de : accroître l'implication des viticulteurs et de leurs organisations dans la $R \& D$, la gestion du vignoble, des outils économiques, la gouvernance de la filière

- Sauvegarder la définition du vin (boisson culturelle originale) et de son ancrage à un territoire : actions syndicales et politiques, communication, lobbying international

- Créer des marques fortes leader dans les appellations, avoir un marketing plus agressif autour des terroirs, communiquer sur les liens aux paysages et à la culture

- Maintenir une réglementation pour garantir les produits, gérer l'accès au foncier et aux ressources, soutenir l'installation des (jeunes) viticulteurs.

\section{b) Analyse des leviers sur l'ensemble des 6 régions}

Sur l'ensemble des 6 régions et des 4 scénarios (Tableau 4), les domaines d'actions se répartissent en proportion équivalente (de $30 \%$ à $26 \%$ ), entre i) la recherche et l'expérimentation (mentionnés par $83 \%$ des participants), ii) les aspects règlementaires, iii) les questions économiques (avant tout lié à la définition et valorisation du vin), iv) l'introduction d'innovation technique ou v) la formation et le conseil (majoritairement aux viticulteurs). Le classement par domaine plus précis d'action, met en avant l'organisation ou la défense des «terroirs», les changements de cépages, ou la qualité du vin. Même si la $R \& D$ ressort en premier, on trouve une diversité de domaines d'action, traduisant l'absence de

\footnotetext{
${ }^{6}$ Organisme de Défense et de Gestion.
} 
Tableau 4. Nombre et \% d'acteurs et de propositions par domaine d'action.

\begin{tabular}{|l|l|l|l|l|}
\hline $\begin{array}{l}\text { Domaine } \\
\text { d'action }\end{array}$ & Nb Acteurs & \%Acteurs & Nb propos & \%propos \\
\hline R\&D & 271 & $\mathbf{8 3}$ & 660 & $\mathbf{3 0}$ \\
\hline Formation & 245 & $\mathbf{7 5}$ & 579 & $\mathbf{2 6}$ \\
\hline Règlement & 157 & $\mathbf{4 8}$ & 288 & $\mathbf{1 3}$ \\
\hline Economique & 279 & $\mathbf{8 5}$ & 747 & $\mathbf{3 4}$ \\
\hline Social & 150 & $\mathbf{4 6}$ & 257 & $\mathbf{1 2}$ \\
\hline Techniques & 260 & $\mathbf{7 9}$ & 662 & $\mathbf{3 0}$ \\
\hline Terroir & 201 & $\mathbf{6 1}$ & 384 & $\mathbf{1 7}$ \\
\hline Environnement & 87 & $\mathbf{2 7}$ & 154 & $\mathbf{7}$ \\
\hline Mod. techno & 157 & $\mathbf{4 8}$ & 306 & $\mathbf{1 4}$ \\
\hline Mod tradit & 226 & $\mathbf{6 9}$ & 656 & $\mathbf{3 0}$ \\
\hline Coexistence & 261 & $\mathbf{8 1}$ & 952 & $\mathbf{4 3}$ \\
\hline Qualité vin & 151 & $\mathbf{4 6}$ & 236 & $\mathbf{1 1}$ \\
\hline Cépages & 122 & $\mathbf{3 7}$ & 179 & $\mathbf{8}$ \\
\hline Irrigation & 69 & $\mathbf{2 1}$ & 95 & $\mathbf{4}$ \\
\hline Enologie & 63 & $\mathbf{1 9}$ & 79 & $\mathbf{4}$ \\
\hline AOC & 101 & $\mathbf{3 1}$ & 160 & $\mathbf{7}$ \\
\hline
\end{tabular}

Tableau 5. Propositions d'action par type/domaine selon l'attitude stratégique sur chaque chemin.

\begin{tabular}{|l|l|l|l|l|l|}
\hline \multirow{2}{*}{$\begin{array}{l}\text { Type et } \\
\text { domaine d'action }\end{array}$} & \multicolumn{4}{|c|}{$\begin{array}{c}\text { \% propositions par type/domaine pour l'attitude dominante de chaque chemin } \\
\text { proactivité positive (PP) ou négative (PN) } 328 \text { participants, 222 actions }\end{array}$} \\
\cline { 2 - 6 } & Conservat PP & Conservat PN & Innovant PP & Nomade PN & Liberal PN \\
\hline Etat & 34 & $\mathbf{5 0}$ & $\mathbf{5 3}$ & 39 & 44 \\
\hline Collectif & 63 & $\mathbf{6 8}$ & $\mathbf{7 1}$ & 63 & 58 \\
\hline Individu & $\mathbf{2 8}$ & 14 & 20 & 13 & 10 \\
\hline Mixte & 27 & $\mathbf{4 0}$ & $\mathbf{4 2}$ & 23 & 23 \\
\hline & & & & & \\
\hline R\&D & 26 & $\mathbf{3 3}$ & $\mathbf{4 6}$ & 16 & 14 \\
\hline Formation & 21 & $\mathbf{3 5}$ & 31 & 26 & 26 \\
\hline Réglementation & 10 & 13 & 16 & 16 & $\mathbf{2 4}$ \\
\hline Economie & 32 & 21 & 23 & $\mathbf{4 5}$ & $\mathbf{5 2}$ \\
\hline Social & 12 & 7 & 8 & $\mathbf{1 7}$ & $\mathbf{2 4}$ \\
\hline Technique & $\mathbf{4 5}$ & 30 & $\mathbf{4 4}$ & 16 & 9 \\
\hline Terroir & 19 & 4 & 7 & $\mathbf{4 2}$ & 21 \\
\hline Environnement & $\mathbf{1 7}$ & 3 & 11 & 4 & 3 \\
\hline Mod technol & 10 & $\mathbf{1 9}$ & $\mathbf{2 4}$ & 8 & 1 \\
\hline Mod traditio & $\mathbf{4 2}$ & 9 & 15 & $\mathbf{5 8}$ & $\mathbf{6 1}$ \\
\hline Coexistence & 32 & $\mathbf{6 0}$ & $\mathbf{5 2}$ & 23 & 22 \\
\hline Qualité Vin & 13 & 5 & 8 & 13 & $\mathbf{1 9}$ \\
\hline Cépages & $\mathbf{1 6}$ & 7 & $\mathbf{1 4}$ & 3 & 0 \\
\hline Irrigation & 4 & 5 & 5 & 4 & 2 \\
\hline Enologie & $\mathbf{7}$ & 3 & 5 & 1 & 3 \\
\hline AOC & 9 & 3 & $\mathbf{1 4}$ & 9 \\
\hline & & 6 & \\
\hline
\end{tabular}

«solution unique» et l'importance de combiner des actions dans des domaines différents. Ces résultats globaux ont été analysés d'une part selon chaque attitude stratégique et d'autre part selon chaque région.

L'analyse des propositions d'action selon les principales attitudes stratégiques (Tableau 5) montre que quelques leviers sont suggérés de manière équivalente pour toutes les stratégies, comme la formation ou les aspects réglementaires, mais que les domaines d'action sont majoritairement discriminants.
- Une forte convergence de ces domaines apparaît tout d'abord entre l'attitude de proactivité positive pour le chemin innovant et celle de proactivité négative sur le chemin conservateur. Les participants mobilisent donc les mêmes domaines d'action dans deux sens différents, pour promouvoir le premier chemin et éviter le second. Les références à l'action publique et à l'action collective y sont les plus affirmés, ainsi que les domaines de R\&D, les aspects techniques (nombreuses propositions précises), avec 
Tableau 6. Propositions d'action par type/domaine selon les régions.

\begin{tabular}{|l|l|l|l|l|l|l|}
\hline Type domaine d'action & Alsace & Bordeaux & Bourgogne & Champagne & Languedoc & Rhône \\
\hline Etat & 59 & $\mathbf{9 6}$ & 82 & 82 & 89 & 83 \\
\hline Collectif & 85 & 98 & 95 & 93 & 92 & 91 \\
\hline Individu & $\mathbf{7 1}$ & 54 & 46 & 49 & 66 & 55 \\
\hline Mixte & 46 & $\mathbf{9 3}$ & 82 & 73 & 77 & 75 \\
\hline Généralités & 49 & 63 & 74 & 67 & 70 & 76 \\
\hline R\&D & 85 & 88 & 87 & 80 & 80 & 80 \\
\hline Formation & 80 & 75 & $\mathbf{8 7}$ & 67 & 73 & 71 \\
\hline Réglementation & 54 & 54 & 41 & 36 & 46 & 52 \\
\hline Economique & 80 & 91 & 95 & 82 & 79 & 85 \\
\hline Social & 56 & 70 & 49 & 40 & 41 & 28 \\
\hline Techniques & 85 & 77 & 74 & 73 & $\mathbf{8 7}$ & 76 \\
\hline Terroir & $\mathbf{6 8}$ & 63 & 59 & 47 & 58 & 66 \\
\hline Environnement & $\mathbf{4 1}$ & 19 & 26 & 16 & $\mathbf{3 8}$ & 20 \\
\hline Mod. technol & 39 & 56 & 54 & 38 & 44 & 53 \\
\hline Mod. tradition & $\mathbf{8 0}$ & $\mathbf{8 1}$ & 59 & 73 & 63 & 61 \\
\hline Coexistence & 56 & 86 & $\mathbf{9 5}$ & 58 & $\mathbf{8 9}$ & 84 \\
\hline Consommateur & 29 & $\mathbf{4 0}$ & 33 & 13 & 24 & 21 \\
\hline Qualité vin & 58 & $\mathbf{6 3}$ & 49 & 42 & 37 & 36 \\
\hline Cépages & $\mathbf{4 6}$ & 40 & 23 & 22 & $\mathbf{4 6}$ & 37 \\
\hline Irrigation & 12 & 14 & 5 & 2 & $\mathbf{4 2}$ & $\mathbf{3 1}$ \\
\hline Enologie & 19 & $\mathbf{2 5}$ & 23 & 11 & 14 & 23 \\
\hline AOC & 32 & 30 & 25 & 38 & 28 & 33 \\
\hline & & & & & \\
\hline
\end{tabular}

en revanche moins de référence aux terroirs et aux traditions, tout en défendant une vision ouverte à la coexistence de plusieurs formes de viticulture.

- Les actions mises en avant pour promouvoir le chemin conservateur se distinguent par le poids des stratégies individuelles et la faiblesse des références à l'action publique nationale (partiellement compensée par les collectivités locales), par des domaines d'action ciblés à la fois sur la défense des «terroirs» et l'environnement, mais aussi l'œnologie (vue comme une variable clé pour ce chemin, sans forcément d'innovation radicale) et les actions visant à «faire accepter les vins marqués par le changement climatique».

- Enfin les attitudes stratégiques visant à repousser la réalisation des scénarios nomade et libéral ont des profils d'action similaires : si les références directes à l'action collective sont moins présentes, c'est surtout la faiblesse des domaines de R\&D et techniques, et d'action sur l'encépagement ou l'environnement qui est observée. La défense du vin, des AOC et des terroirs est mise en avant, dans le cadre d'actions plus politiques.

La comparaison des résultats par région (Tableau 6) montre peu de différence significative sur les grands domaines d'action. Dans les six vignobles, les actions se référant à la $\mathrm{R} \& \mathrm{D}$, à la formation et aux aspects réglementaires sont mentionnées dans des proportions statistiquement proches. Par contre l'examen des types d'action, des enjeux liés à l'environnement ou à des domaines techniques plus précis révèle des préoccupations et options différentes d'une région à l'autre. L'Alsace se distingue ainsi par l'importance de thèmes liés à l'environnement, au choix de cépage avec une vision assez «conservatrice» revendiquant la défense des terroirs ; les acteurs du bordelais sont ceux qui expriment le plus fortement l'importance d'une combinaison entre action publique et collective, avec des domaines de solution qui se réfèrent plus à la qualité des vins, à l'œnologie et à la prise en compte des consommateurs ; la Bourgogne et la Champagne mentionnent moins les questions d'encépagement, d'irrigation ou de réglementation, mais elles se distinguent entre elles par la plus faible mention en Champagne de domaines techniques, liés à l'environnement et au terroir, ou même d'action en direction du consommateur. La vallée du Rhône et le Languedoc ont des profils proches mettant en avant l'irrigation, mais le vignoble languedocien se distingue de son voisin méridional par des références plus fortes à l'environnement et aux innovations techniques.

Les choix d'actions diffèrent donc d'abord selon les attitudes stratégiques, en particulier sur les types d'action et leurs grands domaines, associant soutien au scénario innovant et rejet du conservateur, et en les opposant d'une part à la défense du scénario conservateur et d'autre part aux rejets des scenarios nomades et libéral. Les différences selon les régions sont moins marquées, mais s'expriment dans des leviers plus précis d'action, révélant des impacts et perceptions différents du changement climatique, mais aussi des orientations productives et des formes d'organisations professionnelles différentes selon les régions.

\section{c) Analyse des trajectoires adaptatives}

Il est éclairant d'analyser les leviers d'action en précisant la manière dont se combinent les 6 couples 


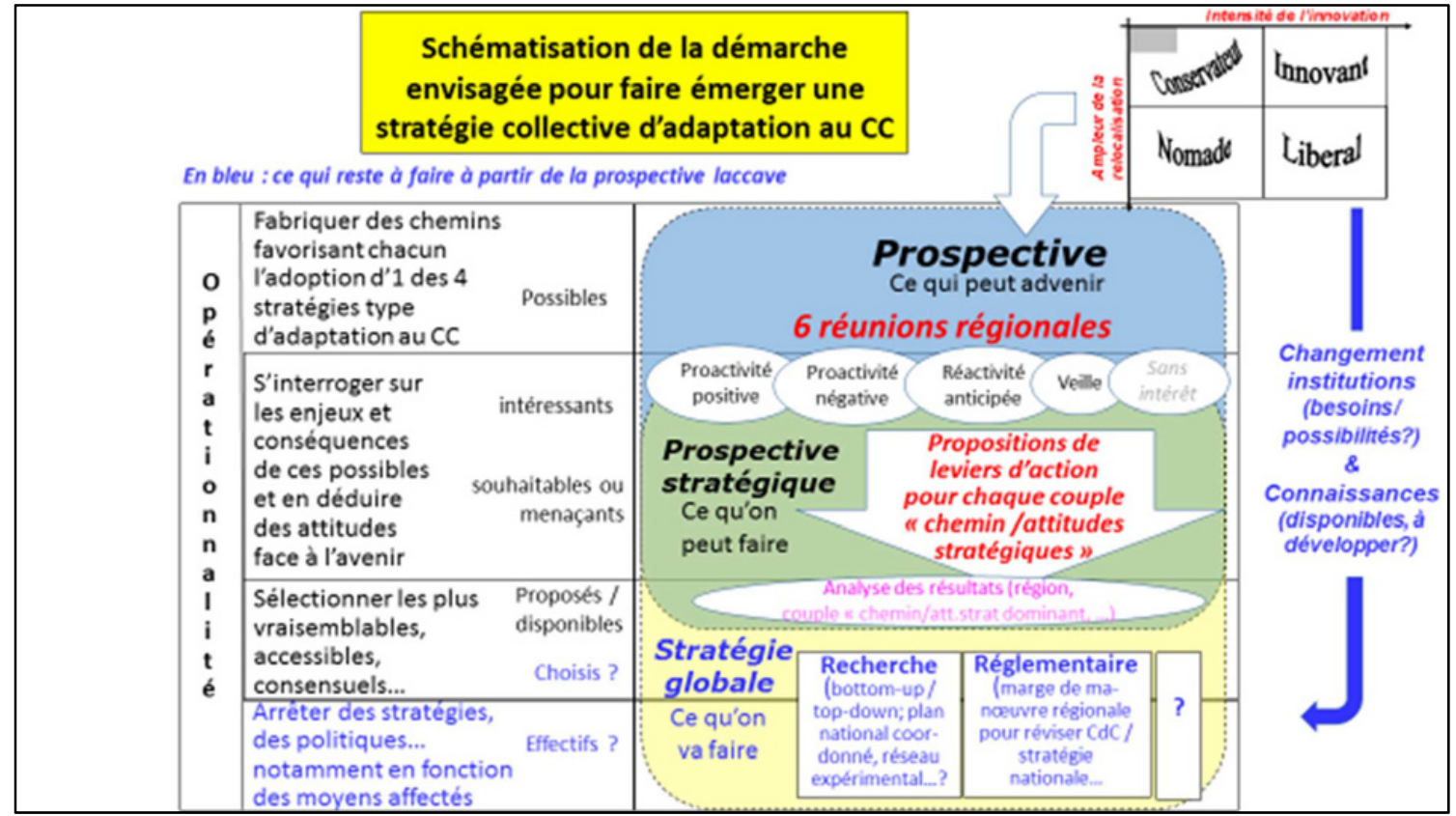

Figure 2. Schématisation de la démarche envisagée pour faire émerger une stratégie collective d'adaptation au changement climatique.

«chemin/attitudes stratégiques» les plus fréquemment choisis dans les votes à savoir :

- Conservateur / réactivité anticipée, (95 sur 342 ou 151 sur 342 si l'on cumule «réactivité anticipée» et «veille» en considérant qu'il s'agit d'une attitude «prudente ou attentiste»)

- Conservateur / proactivité négative (110 sur 342)

- Innovant / proactivité positive (246/342)

- Nomades / proactivité négative (135/342)

- Nomades / prudence ou attentisme (192/342)

- Libéral / proactivité négative (195/342).

Chaque participant s'étant exprimé sur chaque chemin, il est possible d'analyser les différentes combinaisons qu'il porte. Ceci permet d'approfondir la compréhension des différentes trajectoires adaptatives proposées par les participants : Ainsi si l'on s'intéresse au couple «chemin / attitude stratégique» dominant, à savoir le couple «innovant / proactivité positive» (246/342), on se rend compte, au premier niveau de combinaison, que 38 des 246 «innovants proactifs positifs (IPP)» sont dans le même temps «conservateurs proactifs (positifs)», ce qui relativise leur caractère innovateur, alors que 106 de ces IPP sont «conservateurs attentistes» et 92 strictement «non conservateurs». En poursuivant l'analyse de ces combinaisons avec les attitudes stratégiques de ces 246 IPP en matière de «nomadisme» et de «libéralisme», on peut en première analyse, déterminer au sein des IPP, (i) des «innovants à reculons», (ii) des «innovants pour éviter toute délocalisation géographique», (iii) des «innovants intrinsèques».

Ces approfondissements sont en cours, et développés en relation avec le groupe de travail national créé par FranceAgriMer et l'INAO.

\section{Conclusion et perspectives : la suite des travaux pour passer des leviers proposés à l'élaboration d'une stratégie nationale de filière}

Les cinq réunions du groupe de travail national créé par FranceAgriMer et l'INAO conduites à Paris entre janvier 2017 et juillet 2018 ont permis d'avancer aux deux niveaux national et régional :

- au plan national, une démarche de sensibilisation des différentes organisations professionnelles a été effectuée, complétée par un état des lieux sur les évolutions climatiques et leurs impacts. Les instances nationales s'étant approprié le sujet ont été invitées à présenter leurs travaux (APCA, ...). Les instances de gouvernance de la CNAOC et de la Confédération française des IGP ont orienté leurs travaux inter-régionaux sur cette question et invité les prospectivistes de LACCAVE à présenter les travaux en cours lors de leurs AG.

- au plan régional, les représentants des différents vignobles ont été informés des résultats des exercices de prospective régionale, et des analyses ; ils ont été invités à provoquer des réunions dans leurs bassins respectifs afin d'y élaborer des trajectoires d'adaptation, et à partager ensuite lors des réunions centrales ces actions, envisagées à terme ou en cours de réalisation.

\section{1) Objectifs : vers la définition d'une stratégie nationale :}

Ducôté de la Recherche scientifique, il a été décidé (i) de prolonger les travaux interdisciplinaires dans le cadre du projet LACCAVE2.21, ii) de valoriser la démarche dans les communautés scientifiques et de l'adaptation au 
changement climatique avec l'appui européen de la KIC Climat (plateforme www. Agrisource.org) et (iii) afin de favoriser la diffusion et les échanges avec les organisations professionnelles, d'élaborer un document synthétique de sensibilisation au changement climatique, sous la forme d'infographies réalisées par l'INRA.

Du côté de la filière viticole, l'objectif général consiste en l'inclusion de l'exercice prospectif et des propositions qui en sont issues dans une démarche visant à l'émergence d'une stratégie collective d'adaptation de la filière vignes et vins au changement climatique à l'échelle de la France. Les acteurs désireux d'anticiper les conséquences du changement climatique et de maitriser au mieux leur avenir, ont confirmé la nécessité d'élaborer une telle stratégie à l'échelle nationale, notamment en s'appuyant sur les travaux de prospective, qui mettent à disposition :

- les différentes approches et les résultats recueillis en région, notamment les «attitudes stratégiques» majoritairement retenues par les acteurs, et les leviers d'action proposés ;

- les représentations du problème fournies par les scientifiques ;

- les propositions de priorités en matière de recherche.

On observe qu'il ne s'agit pas là de privilégier un "scénario-cible". Au contraire la stratégie globale retenue devra combiner des actions satisfaisant conjointement plusieurs attitudes stratégiques correspondant respectivement aux 4 «chemins».

La stratégie nationale devra présenter une cohérence globale et prendre en compte des adaptations spécifiques aux différentes régions. Elle sera présentée au Conseil Spécialisé de FranceAgriMer et aux Comités Nationaux AOC et IGP de l'INAO pour être améliorée et validée. Sur la forme, elle comportera un plan d'actions avec des points de vigilance et idéalement des indicateurs clés de réussite et d'échec. La question de décliner cette stratégie également par segments de la filière (AOC ; IGP, VSIG) sera également envisagée.

\section{2) Méthodologie d'élaboration de la stratégie nationale}

Il a été considéré qu'une méthode de type «topdown»risquerait de négliger les spécificités régionales et la variabilité naturelle et culturelle entre bassins ; à l'opposé, une méthode plus inductive de type «bottomup»supposerait une mobilisation assez forte et homogène entre les différents bassins. C'est une méthodologie qui combine les deux approches qui a été retenue, qui favorise des allers-retours successifs entre niveaux régional et national. Il s'agit à ce stade de pistes de travail à valider par le groupe de travail commun «FranceAgriMer-INAO».

Il a ainsi été proposé (ordre du jour de la réunion du groupe de travail du 9 octobre) :

- une présentation des propositions auprès des instances décisionnelles de FAM et de l'INAO, Conseil spécialisé et Comités nationaux des AOC d'une part et des IGP d'autre part,

- l'organisation d'un séminaire national avec présentations des propositions régionales et validation de la méthode pour un plan d'action climat en viticulture (janvier 2019),
- un retour dans chaque région en 2019, avec l'objectif :

(i) d'indiquer les priorités telles que perçues par la Recherche,

(ii) d'élaborer actions et leviers régionaux

- un travail de compilation au plan national afin de :

(iii) vérifier les cohérences inter-régionales,

(iv) en déduire un cadre commun au plan national.

A ces deux niveaux, seront analysées particulièrement, (i) d'une part les actions concernant les thématiques de recherche à approfondir (sélection variétale, système de production et pratiques culturales, process de vinification, marché et attentes des consommateurs,....) (ii) d'autre part les aspects touchant à l'organisation et la gouvernance de la filière qui mériteraient d'évoluer (réglementation, mesures OCM, PAC, ...), enfin (iii) les mesures orientées vers la mitigation (mesures d'atténuation d'émission des GES, stockage de $\mathrm{C}$ dans les sols, ... ). Enfin, les modalités de communication/diffusion de la stratégie nationale restent à préciser.

\section{3) La poursuite des travaux :}

Les six réunions régionales ${ }^{7}$ de restitution participative de la prospective LACCAVE ont fourni un matériau riche et important. Bien qu'issu d'une population impliquée mais non représentative au sens statistique de l'ensemble des maillons de la filière viti-vinicole française, il mérite d'être analysé en profondeur afin de servir utilement l'émergence d'une stratégie collective d'adaptation au changement climatique. Plusieurs pistes ont été identifiées dans ce sens.

Ont ainsi été consignées les «attitudes stratégiques» adoptées par les 342 participants à ces réunions, et leurs propositions de leviers d'action - plus de 2200 - pour l'ensemble des couples «chemin favorisant l'adoption d'un des 4 scénarios d'adaptation au changement climatique $\mathrm{X}$ attitude stratégique». Sont ici privilégiées les pistes permettant de passer «pas à pas» de la prospective (ce qui peut advenir) à la prospective stratégique (ce que l'on peut faire) à la stratégie (ce que l'on va faire) en visant à :

- approfondir l'analyse des leviers d'action en examinant la variabilité régionale des résultats (cf. III- 4-b)

- approfondir la compréhension des trajectoires adaptatives proposées par les participants (III-4-c)

- utiliser ces analyses pour tenter de faire émerger la meilleure organisation possible visant à la fois :

- un cadre institutionnel national, comprenant éventuellement des modalités par segment

- une adaptabilité aux différentes échelles territoriales (compte tenu notamment de la variabilité des effets du CC sur vigne en fonction des régions)

et ce, en jouant sur au moins deux leviers :

- des directions de recherche, voire l'émergence d'un plan national à l'instar de celui à l'œuvre sur le dépérissement

${ }^{7} \mathrm{NB}$ : une septième réunion de restitution régionale est en préparation pour la région Val de Loire. 
- des évolutions «juridico-institutionnelles»permettant d'accroître par exemple l'adaptabilité des règles et des objets de révision des cahiers des charges des signes d'identification de la Qualité et de l'Origine (SIQO), AOP, IGP mais aussi de la règlementation de l'Agriculture Biologique.

\section{Références}

[1] P. Aigrain, B. Bois, P. Brugière, E. Duchène, I. Garcia de Cortazar, E. Giraud-Heraud, J. Gautier, H. Hannin, N. Ollat, JM. Touzard, 2016a. Lessons from a Prospective Study on the French wine industry under climate change, in Ollat N, Touzard J.M., Garcia de Cortazar-Atauri I., Quenol H., Van Leeuwen C., (Eds), Sustainable grape and Wine production under climate change, Bordeaux : Vigne et Vin publications Internationales, pp. 253-263

[2] P. Aigrain, F. Brugière, E. Duchêne, I. Garcia De Cortazar Atauri, J. Gautier, E. Giraud-Heraud, H. Hannin, N. Ollat, J.-M. Touzard (2016b). Adaptation au changement climatique : intérêt d'une démarche prospective. BIO Web of Conferences 7
[3] P. Aigrain, F. Brugière, E. Duchêne, I. Garcia De Cortazar Atauri, J. Gautier, E. Giraud-Heraud, H. Hannin, N. Ollat, J.-M. Touzard, 2016c. Travaux de prospective sur l'adaptation de la viticulture au changement climatique : quelles séries d'événements pourraient favoriser différentes stratégies d'adaptation? BIO Web of Conferences 7

[4] P. Aigrain, F. Brugière, E. Duchène, J. Gautier, E. Giraud Heraud, H. Hannin, I. Garcia de Cortazar, N. Ollat, JM Touzard, (2016d). Une prospective pour le secteur vigne et vin dans le contexte du changement climatique. Les synthèses de FranceAgrimer, $\mathrm{n}^{\circ} 40$, septembre 2016

[5] R. Few, K. Brown, E. Tompkins, Clim. Policy 7, 45 (2011)

[6] M. Godet, P. Durance, La prospective stratégique pour les entreprises et les territoires (Dunod, Paris, 2011)

[7] G.V. Jones, L.B. Webb, J. Wine Res. 21, 103 (2010)

[8] N. Ollat, J.-M. Touzard, J. Int. des Sci. de la Vigne et du Vin, 1 (2014)

[9] N. Ollat, J.-M. Touzard, C. Van Leeuwen, J. Wine Econ. 11, 1 (2016) 